\title{
The itramyocardial stress and the mechanics of the left ventricular contraction
}

\author{
R.M. Shoucri \\ Department of Mathematics and Computer Science \\ Royal Military College of Canada \\ Kingston, Ontario, Canada, $k 7 k 7 b 4$.
}

\begin{abstract}
A mathematical model has been developed in which the force generated by the fibres of the myocardium is represented as force/unit volume of the myocardium. By using this approach, new indexes have been derived that can be used to express the ventriculo-arterial coupling and to differentiate between various cardiomyopathies. It has also been possible to derive a mathematical expression for the intramyocardial stress induced in the passive medium of the myocardium as the sum of two components, one component induced by the intraventricular pressure and the other component induced by the force/unit volume of the myocardium representing the fibres force. Relations between the intramyocardial stress and the parameters describing the end-systolic pressurevolume relation (espvr) are presented and discussed.
\end{abstract}

\section{Introduction}

The study of the relation between the stress induced in the myocardium and the intraventricular pressure has been known as the law of Laplace [1-4], it was initially derived by using the linear theory of elasticity for a passive inert medium. When it comes to an active medium like the myocardium, one of the difficult problems in cardiac mechanics is the study of the relation between myocardial fibre stress and the fibre orientation on the one hand and the parameters describing the performance of the right or left ventricle as a pump on the other hand. There are basically two general approaches for this problem. 
The first approach consists in developing models for the fibre orientation in the myocardium, as well as for the active force generated by the fibre according to the Huxley cross-bridge model for instance. The studies by Waldman et al [5] and Vendelin et al [6] follow this approach. Although this approach is important at the fundamental research level in that it gives more insight into the intricate relations between the different parameters involved, its application at the clinical level is more difficult.

The second approach, which has been developed in a series of previous studies [7-13], consists in modeling the active force generated by the myocardial fibres as force/unit volume of the myocardium. This approach is well known in the theory of elasticity. In view of the complex structure of the myocardium, one can confidently assume that the total intramyocardial stress $\sigma_{t o t}$ induced in the passive medium of the myocardium is a tensorial quantity with six components (3 normal and 3 shear stresses) [14]. Under the assumption of a symmetrical contraction of the myocardium, the intramyocardial stress $\sigma_{t o t}$ reduces to a vectorial quantity with three orthogonal components (for instance in a cylindrical coordinate system we have radial $\sigma_{r}$, circumferential $\sigma_{c}$, and longitudinal $\sigma_{L}$ components).

This second approach has the advantage that it splits the complexity of the problem in two parts: 1) a study of the relation between the force generated by the myocardial fibres in the complex structure of the myocardium and the force/unit volume of the myocardium used in the modeling process; 2) a study of the relation between the force/unit volume and the mechanics of cardiac contraction. Consequently one does not need to start by step (1) before moving to step (2), one can directly start with step (2) if the interest is directly in the study of the mechanics of cardiac contraction. This is a great simplification of the problem, when step (2) has been solved one can move back to step (1) if required. Results obtained by using this approach and related to clinical applications of the end-systolic pressure-volume relation (espvr), the ventriculoarterial coupling, and the calculation of the stress induced in the passive medium of the myocardium are discussed in this study. We shall confine our attention to the left ventricle, but the approach used can also be applied in a similar way to the right ventricle [15].

\section{Mathematical model}

\subsection{Pressure-volume relation}

A thick-walled cylinder contracting symmetrically is used to represent the left ventricle (see Figure 1, left), the cylindrical model is known to be a good approximate representation of the ventricles near the equatorial region. Transverse isotropy around the cylinder axis is assumed. A myocardial fibre is assumed to have a helical shape, with a variable inclination angle as we move from the endocardium to the pericardium. The projection of a helical fibre on the cross-section of the myocardium appears as the dotted circle shown in Figure 1 


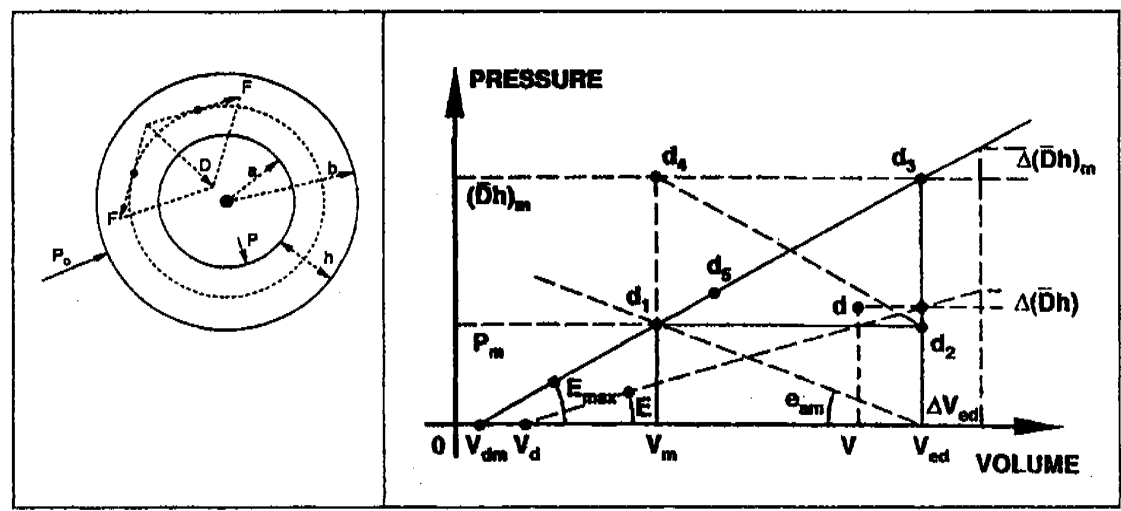

Figure 1 (left): cross-section of a thick-walled cylinder representing the myocardium. A helical muscular fibre will be projected on the cross-section of the myocardium as shown by the dotted circle, $D=$ force/unit volume of the myocardium. $P$ is the left ventricular cavity volume, $P_{o}$ is the outer pressure on the pericardium, $a=$ inner radius, $b=$ outer radius, $h=b-a=$ thickness of the myocardium.

(right): simplified drawing showing the end-systolic pressure-volume relation (espvr) represented by the line $d_{3} V_{d m}$ with slope $E_{\max }=$ left ventricular elastance. $\overline{D h}=$ radial active force/unit area generated on the inner surface of the myocardium (radial fibre stress at the endocardium). The point $d_{5}$ is the middle point of the segment $d_{3} V_{d m}$. During a normal ejecting contraction, the point $d$ of coordinates $(\bar{D} h, V)$ moves near the line $d_{2} d_{4}$. The pressure-volume loop during a normal ejecting cycle is represented by $V_{e d} d_{2} d_{1} V_{m}$. It is assumed that $V_{m} \approx V_{e s}$ the end-systolic volume. The change $\Delta(\bar{D} h)$ corresponds to $\Delta V_{e d}$ according to the Frank-Starling mechanism.

(left). Because of the assumed symmetry of the problem, the myocardial fibres generate a radial active force $D$ per unit volume of the myocardium. The radial active force/unit area on the endocardium (radial fibre stress at the endocardium) is given by $\int_{a}^{b} D d r=\bar{D} h$, where $\bar{D}$ is an average value calculated by the mean valuetheorem, $h=b-a=$ thickness of the myocardium, $a=$ inner radius, $b=$ outer radius.

The relation between the forces acting on the myocardium and the change in cavity volume has been derived by using the theory of large elastic deformation [7] in the case of a quasi-static approximation (inertia forces and viscous forces neglected). Without entering into the mathematical details, one can intuitively write the equilibrium of the radial forces/unit area at the endocardium in the form

$$
\bar{D} h-\left(P-P_{\alpha}\right) \approx E\left(V_{e d}-V\right)
$$

Near end-systole when $E$ reaches its maximum value $E_{\max }$ (see Figure 1, right) 


$$
(\bar{D} h)_{m}-\left(P_{m}-P_{o m}\right)=E_{\max }\left(V_{e d}-V_{m}\right)
$$

$V=\pi a^{2} L$ is the cavity volume, $V_{e d}$ is the cavity volume at end-diastole (when $d V / d t=0$ ), and $E$ is the elastance coefficient. The index $m$ is used to indicate the variables involved when $E$ reaches $E_{\max }$, which is supposed to represent the maximum state of activation of the myocardium. We shall assume in our calculations that $V_{m} \approx V_{e s}$ the end-systolic volume (when $d V / d t=0$ ). The right hand side of eqns (1) is equivalent to the force resulting from the elastic deformation of the passive medium of the myocardium.

Eqn (1b) (and similarly eqn (1a)) can be looked at in three ways (see Figure 1, right) as explained in the following :

1) If $(\bar{D} h)_{m}$ is kept constant and $P_{m}$ and $V_{m}$ are varied, as if a balloon is blown against a constant $(\bar{D} h)_{m}$, the pressure-volume relation is given in a simplied way by the end-systolic pressure-volume relation (espvr) $d_{3} V_{d m}$.

2) In an isovolumic contraction (non-ejecting contraction) at constant $V_{e d}$, the maximum isovolumic pressure generated by the myocardium is $(\overline{D h})_{m}$ and is indicated by the point $d_{3}$ in Figure 1 (right).

3) During an ejecting contraction, the pressure-volume relation is given in a simplified way by the loop $V_{e d} d_{2} d_{l} V_{m}$. In this case $\bar{D} h, P, V$ and $E$ are varying simultaneously during the cardiac cycle, and $\bar{D} h$ reaches $(\bar{D} h)_{m}$ at point $d_{4}$ in Figure 1 (right).

These three aspects of the pressure-volume relation are summarized by eqns (1). Equs (1) can be split as follows

$$
\begin{aligned}
& \bar{D} h=E\left(V_{e d}-V_{d}\right) \\
& (\bar{D} h)_{m}=E_{\max }\left(V_{e d}-V_{d m}\right) \\
& P-P_{o}=E\left(V-V_{d}\right) \\
& P_{m}-P_{o m}=E_{\max }\left(V_{m}-V_{d m}\right)
\end{aligned}
$$

$V_{d}$ and $V_{d m}$ are shown in Figure 1 (right). Eqns (3) represent the way the pressurevolume relation was first introduced. From eqns (2) one can see the direct relation between the elastance $E$ and the active state of the myocardium represented by $\bar{D} h$, the radial active force/unit area generated on the endocardium (radial fibre stress at the endocardium).

\subsection{Intramyocardial stress calculation}

By using the linear theory of elasticity one can also derive the same eqns (1) previously obtained by using the theory of large elastic deformation [7], the difference between the linear theory and the theory of large elastic deformation is in the way the elastance coefficient $E$ is expressed in terms of the parameters describing the elastic properties of the myocardium. But this point is of minor importance for experimentalists who use to calculate $E$ directly from experimental measurements. Consequently it is the theory of linear elasticity that we have used to calculate the intramyocardial stress induced in the passive 
medium of the myocardium by using the mathematical formalism described.

Detailed mathematical analysis for the calculation of the radial $\sigma_{r}$, circumferential $\sigma_{c}$ and longitudinal $\sigma_{L}$ components of the total intramyocardial stress $\sigma_{t o t}^{2}=\sigma_{r}^{2}+\sigma_{c}^{2}+\sigma_{L}^{2}$ induced in the passive medium of the myocardium have been published elsewhere $[12,13]$. We shall confine our attention here to some relations that illustrate the link between the circumferential stress at the endocardium $\sigma_{c a}$ and the parameters describing the pressure-volume relation. The circumferential stress represents nearly $90 \%$ of the total stress induced in the passive medium of the myocardium, it can be considered as a good approximation to study the state of the stress in the myocardium. We have the following relations (see $[12,13]$ for details)

$$
\left(\sigma_{c a}\right)_{m}\left(V_{\omega} /\left(2 V_{m}+V_{\omega}\right)\right)=\left(P_{m}-P_{o m}\right)-(\bar{D} h)_{m}
$$

which can be split in two equations

$$
\begin{aligned}
& \left(\sigma_{c a}\right)_{d m}\left(V_{\omega} /\left(2 V_{m}+V_{\omega}\right)\right) \approx-(\overline{D h})_{m} \\
& \left(\sigma_{c a}\right)_{p m}\left(V_{\omega} /\left(2 V_{m}+V_{\omega}\right)\right) \approx P_{m}-P_{o m}
\end{aligned}
$$

The suffix $m$ indicates as usual that the calculations to be discussed later on are done when $E=E_{\max }$ (it has been dropped in references [12,13] for simplicity, but we keep it here to be consistent with the notation used). Note how the splitting of $\left(\sigma_{c a}\right)_{m}=\left(\sigma_{c a}\right)_{d m}+\left(\sigma_{c a}\right)_{p m}$ corresponds to the splitting of $V_{e d}-V_{m}$ into $V_{e d}-V_{d m}$ and $V_{m}-V_{d m}$ in eqns.(2),(3). $V_{\omega}=\pi\left(b^{2}-a^{2}\right) L$ is the volume of the myocardium and it is supposed to be constant for an incompressible medium. By comparing eqns.(4) with eqns.(2) and (3) one can see how the ventricular elastance $E$ can be directly related to the stress induced in the passive medium of the myocardium, an idea that was discussed qualitatively by Westerhof [16]. During the systolic phase the induced stress $\sigma_{c a}$ is negative (compression) because $\bar{D} h>P$.

It is to be noted that by neglecting $(\overline{D h})_{m}$ the stress is calculated only by using eqn (4c), which explains the apparent contradictions reported in the literature between measured and calculated stress [17-19].

In the following, the calculations were carried out by assuming that the pressure at the pericardium $P_{o} \approx 0$.

\section{Results}

\subsection{The ventriculo-arterial coupling}

The ratio $E_{m a x} / e_{a m}$ (maximum ventricular elastance/corresponding arterial elastance, see Figure 1, right) can be used to distinguish between different states of the myocardium. The stroke work $S W \approx$ area $V_{e d} d_{2} d_{l} V_{m}$ represents the energy delivered to the systemic circulation. When $d_{l}$ moves along the espvr represented in a simplified way in Figure 1 (right) by the line $d_{3} V_{d m}, S W$ reaches its maximum value $(S W)_{\max }$ when $d_{l}$ coincides with $d_{5}$ (the middle point of the segment $d_{3} V_{d m}$ ). 

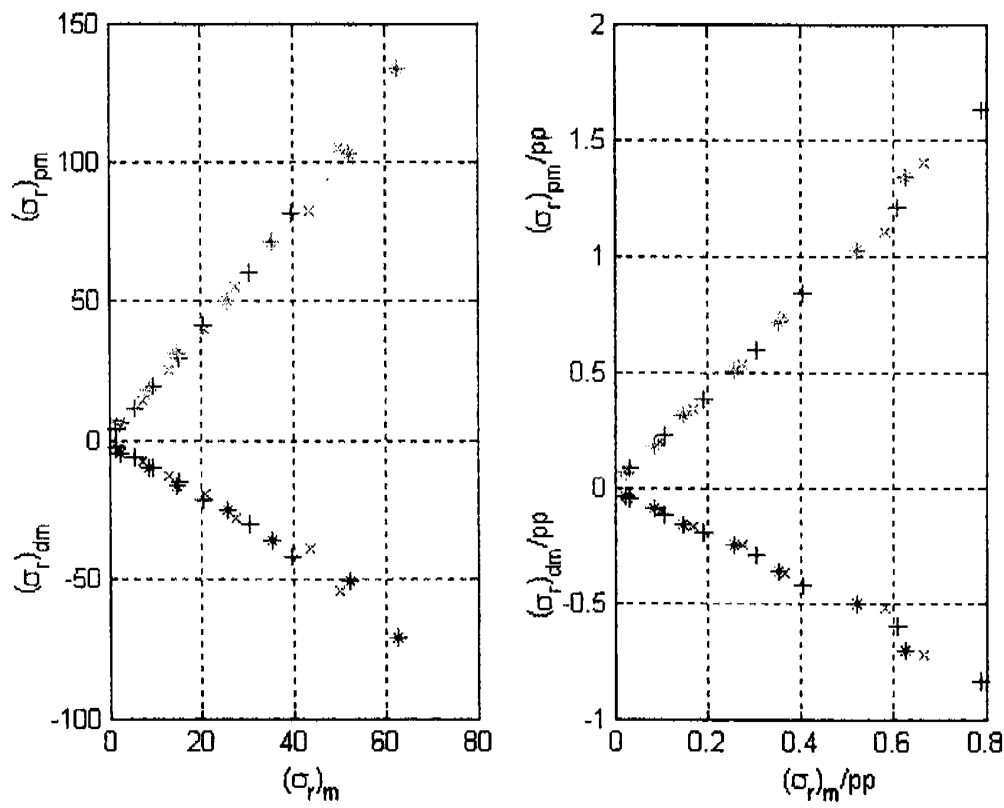

Figure 2 (left): the abscissa $\left(\sigma_{r}\right)_{m}=\left(\sigma_{r}\right)_{p m}+\left(\sigma_{r}\right)_{d m}$ is the radial stress induced in the passive medium of the myocardium calculated at different depths from the pericardium (left) to the endocardium (right), the ordinates are the components $\left(\sigma_{r}\right)_{p m}$ induced by $P_{m}$ and $\left(\sigma_{r}\right)_{d m}$ induced by $(\vec{D} h)_{m}$. The calculation is carried out for three perfusion pressures $\mathrm{pp},+50 \mathrm{mmHg}$, $x 75 \mathrm{mmHg}, * 100 \mathrm{mmHg}$.

(right): same as the left hand side, with the values of the stresses divided by the perfusion pressures pp.

Calculation based on experimental data taken from ref. [22].

One can consequently observe that in the normal physiological state of the myocardium (maximum efficiency for oxygen consumption by the myocardium $[9,10,20,21]) E_{\max } / e_{a m} \approx 2$ which corresponds to $P_{m} /(\overrightarrow{D h})_{m} \approx 1 / 3\left(d_{l}\right.$ below $d_{5}$ on the segment $\left.d_{3} V_{d m}\right)$; for mildly depressed state of the myocardium one has $E_{\max } / e_{a m} \approx 1$ which corresponds to $P_{m} /(\bar{D} h)_{m} \approx I$ (d $d_{1}$ and $d_{5}$ coincides); for severely depressed state of the ventricle one has $E_{\max } / e_{a m}<1\left(d_{1}\right.$ above $d_{5}$, in this region an increase in $P_{m}$ results in a decrease of the stroke work $S W$ ). Experimental results that confirm these calculations have been published [20, 21]. By introducing of the active force/unit area $\bar{D} h$ in the formalism describing the pressure-volume relation, the espvr offers a simple way to characterize the state of the myocardium. The areas under the espvr can also be used to differentiate between different cardiomyopathies $[8,11]$. 

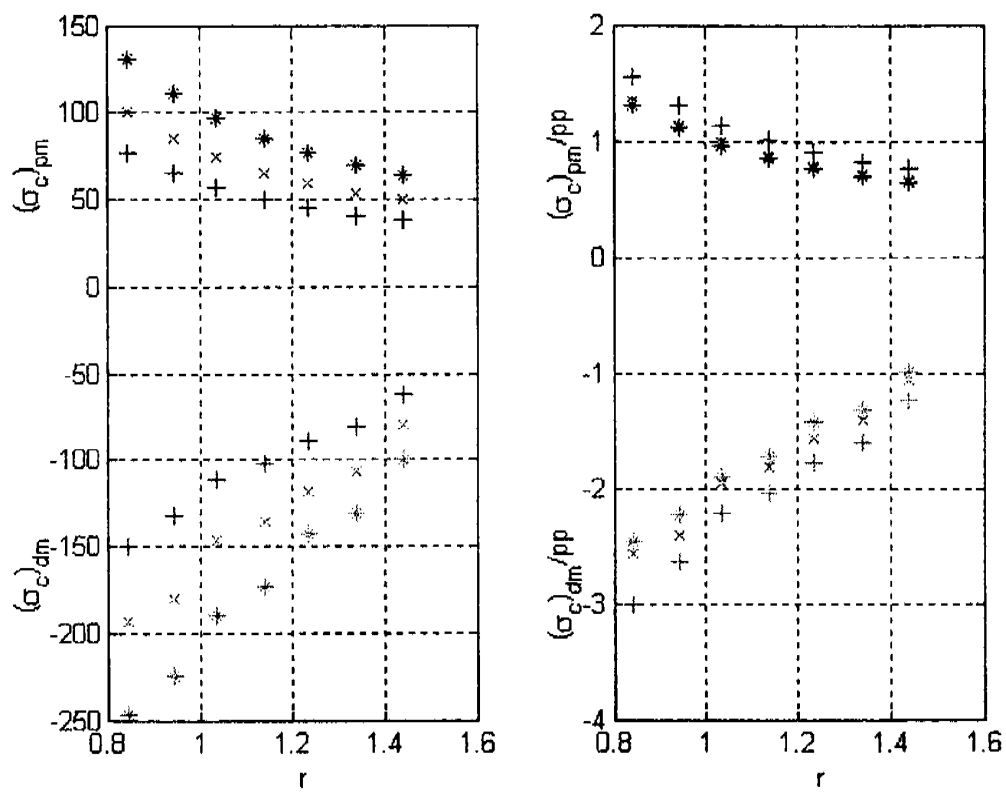

Figure 3 (left): the abscissa is the radial distance $\mathrm{r}$ in $\mathrm{cm}$ from the endocardium $(r=0.8 \mathrm{~cm})$ to the pericardium $(r=1.6 \mathrm{~cm})$, the ordinates are the circumferential stress components $\left(\sigma_{c}\right)_{p m}$ induced by $P_{m}$ and $\left(\sigma_{c}\right)_{d m}$ induced by $(\overrightarrow{D h})_{m}$. The calculation is carried out for three different perfusion pressures pp, $+50 \mathrm{mmHg}, \mathrm{x} 75 \mathrm{mmHg}, * 100 \mathrm{mmHg}$.

(right): same as the left side, with the values of the stresses divided by the perfusion pressures pp.

Calculation based on experimental data taken from ref. [22].

\subsection{Intramyocardial stress calculation}

Experimental verification of the mathematical formalism discussed in section 2 to calculate the intramyocaldial stress is based on data published by Mihailescu et al [22], and Burns et al [18]. The important point to note is the consistency of the results obtained for two independent experiments and based on the theory of linear elasticity, whereas previous results have indicated that the linear theory of elasticity gives inconsistent results [17,19]. In the experiment reported by Mihailescu et al [22] the experiment was carried out on excised hearts of adult cats, and the stress in the myocardium was measured by micropipette transducers and a controlled displacement of those transducers inside the myocardium. One can assume that the micropipette will sense the total force acting on it, and if properly calibrated it will measure the total intramyocardial stress induced in the passive medium of the myocardium $\left(\sigma_{t o t}\right)_{m}=\left(\sigma_{t o}\right)_{p m}+\left(\sigma_{t o t}\right)_{d m}$. From $\left(\sigma_{t o t}\right)_{m}$ one can calculate $P_{m}-(\bar{D} h)_{m}$ and by measuring $P_{m}$ one can calculate $(\bar{D} h)_{m}$. One 

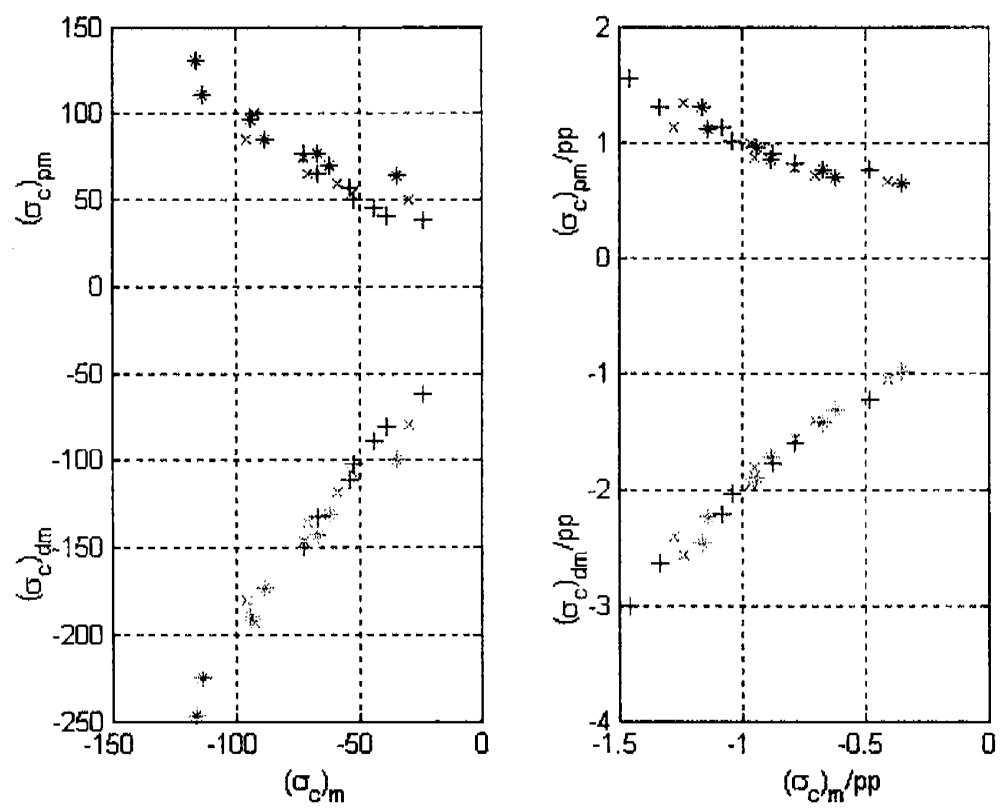

Figure 4 (left): the abscissa $\left(\sigma_{c}\right)_{m}=\left(\sigma_{c}\right)_{p m}+\left(\sigma_{c}\right)_{d m}$ is the circumferential stress induced in the passive medium of the myocardium calculated at different depths from the pericardium (right) to the endocardium (left), the ordinates are the components $\left(\sigma_{c}\right)_{p m}$ induced by $P_{m}$ and $\left(\sigma_{c}\right)_{d m}$ induced by $(\bar{D} h)_{m}$. The calculation is carried out for three different perfusion pressures pp, $+50 \mathrm{mmHg}, \times 75 \mathrm{mmHg}$, * $100 \mathrm{mmHg}$.

(right): same as the left side, with the values of the stresses divided by the perfusion pressures pp.

Calculation based on experimental data taken from ref. [22].

can then calculate the components $\left(\sigma_{t o}\right)_{p m}$, and $\left(\sigma_{t o t}\right)_{d m}$. Finally the components $\left(\sigma_{r}\right)_{p m},\left(\sigma_{c}\right)_{p m},\left(\sigma_{L}\right)_{p m}$ and $\left(\sigma_{r}\right)_{d m s}\left(\sigma_{\partial}\right)_{d m},\left(\sigma_{L}\right)_{d m}$ can be calculated according to the procedure outlined in reference [12]. Figure 2 shows the radial stress $\left(\sigma_{r}\right)_{m}=$ $\left(\sigma_{r}\right)_{p m}+\left(\sigma_{r}\right)_{d m}$ induced in the passive medium of the myocardium and calculated in this way, the ordinates are the components $\left(\sigma_{r}\right)_{p m}$ and $\left(\sigma_{r}\right)_{d m}$ (the suffix $m$ was dropped in references [12,13] for simplicity, it is kept here for consistency in the notation). The results of Figure 2 corresponds to data calculated at different depths in the myocardium for three different perfusion pressures $\mathrm{pp}$, from pericardium (left) to endocardium (right). Similar results for the circumferential stress $\left(\sigma_{c}\right)_{m}=\left(\sigma_{c}\right)_{p m}+\left(\sigma_{c}\right)_{d m}$ are shown in Figure 4, the ordinates are the components $\left(\sigma_{c}\right)_{d m}$ and $\left(\sigma_{c}\right)_{p m}$. Note that during the systolic phase $\sigma_{c}$ is negative ( $\bar{D} h>P$ ), consequently the pericardium is to the right side of Figure 4 and the endocardium to the left side. Figure 3 shows the radial variation of the 

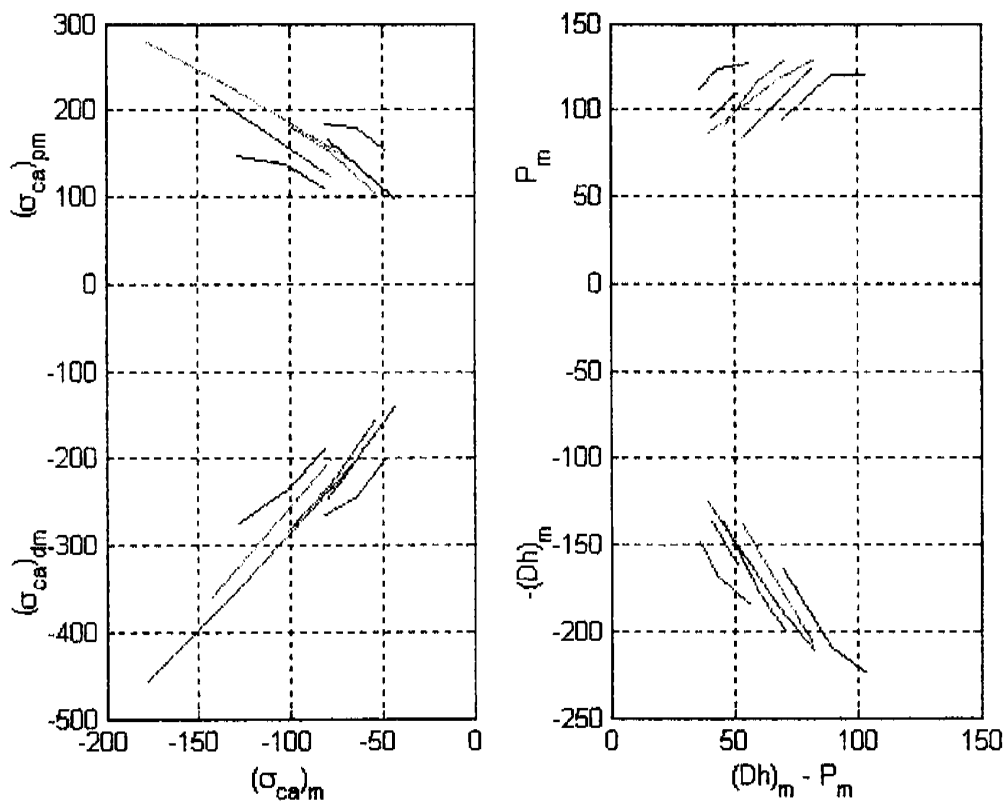

Figure 5 (left): the abscissa $\left(\sigma_{c a}\right)_{m}=\left(\sigma_{c a}\right)_{p m}+\left(\sigma_{c a}\right)_{d m}$ is the circumferential stress induced in the passive medium of the myocardium at the endocardium (at $r=a$ ). The ordinates are the components $\left(\sigma_{c}\right)_{p m}$ induced by $P_{m}$ and $\left(\sigma_{c}\right)_{d m}$ induced by $(\bar{D} h)_{m}$. Results from experiments on 6 dogs. (right): values of $P_{m}$ and $-(\bar{D} h)_{m}$ drawn against $(\bar{D} h)_{m}-P_{m}$ correspond to the circumferential intramyocardial stresses calculated on the left side.

Calculation based on experimental data taken from ref. [18].

circumferential stress components $\left(\sigma_{c}\right)_{d m}$ and $\left(\sigma_{c}\right)_{p m}$, which are large near the endocardium $(r=0.8 \mathrm{~cm})$ and decreases towards the pericardium $(r=1.6 \mathrm{~cm})$. One can note from the results of Figures 2 and 4 as well as the results published in reference [12] that we have the relations $\left(\sigma_{t o}\right)_{d m} /\left(\sigma_{t o}\right)_{p m} \approx\left(\sigma_{c}\right)_{d m} /\left(\sigma_{c}\right)_{p m} \approx$ $\left(\sigma_{r}\right)_{d m} /\left(\sigma_{r}\right)_{p m}=-(\bar{D} h)_{m} / P_{m}$. These relations can be related to $E_{\max }$ by using the triangular relations shown in Figure 1 (right).

Further evidence for the consistency of these results has been obtained from the experimental data published by Burns et al [18]. The experiments in this case were carried out on mongrel dogs with the heart exposed, the circumferential stress indicated $P M_{\sigma l}$ in Table 1 of Burns et al [18] is directly measured by using auxotonic force gauges. This measurement has been approximated by $\left(\sigma_{c a}\right)_{m}$ in our notation, on the basis that the gauge will sense the maximum circumferential stress acting at the endocardium with $r=a=$ inner radius of the myocardium. The procedure for the calculation of $\left(\sigma_{c a}\right)_{p m}$ and $\left(\sigma_{c a}\right)_{d m}$ is reported in reference [13], the results are shown in Figure 5 (left), the right hand-side of Figure 5 
shows the corresponding values of $P_{m}$ and $-(\bar{D} h)_{m}$ drawn against $(\bar{D} h)_{m}-P_{m}$ and calculated by using eqns (4). One should observe the similarity between the curves of Figure 5 (left) obtained from experiments on 6 dogs and calculated at the endocardium, and the result of Figure 4 obtained from an experiment on a cat at three different perfusion pressures and at different depths in the myocardium. This similarity gives further evidence for the consistency of the mathematical formalism used.

\section{Conclusion}

This study has presented some results based on the linear theory of elasticity on the way to calculate the intramyocardial stress as the sum of two components, a component induced by the intraventricular pressure and a component induced by the fibres force represented as force/unit volume of the myocardium. The formalism developed has the interesting feature that it can be linked to the espvr and to the parameters describing the mechanics of ventricular contraction. Possible clinical applications of these results in cases of hypertrophy or hypertension in the future may produce interesting results.

\section{References}

[1] Moriarty, J.F., The law of Laplace, its limitations as a relation for diastolic pressure, volume, or wall stress of the left ventricle, Circulation Research 46, pp. 321-331, 1980.

[2] Li, K.-J.J., A new approach to the analysis of cardiovascular function: allometry (Chapter 2). Analysis and assessment of cardiovascular function, eds. G.M. Drzewiecki \& K.-J.J. Li, Springer Verlag, New York, pp. 13-29, 1995.

[3] Verdonck, P., Vierendels, J. \& Dierickx, P., Cardiac mechanical models (Chapter 1). Intra and extra corporal cardiovascular fluid dynamics, (vol.1), ed. P. Verdonck, WIT Press, United Kingdom, pp. 1-49, 1998.

[4] Rabbany, S.Y., Kresh, J.Y. \& Noordergraaf, A., Myocardial wall stress: evaluation and management, Cardiovascular Engineering 5, pp. 3-10, 2000.

[5] Waldman, L.K., Nosan, D., Francesco, V. \& Covell, J.W., Relation between transmural deformation and local myofiber direction in canine left ventricle, Circulation Research 63, pp. 550-562, 1988

[6] Vendelin, M., Bovendeerd, P.H.M., Engelbrecht, J. \& Arts, T., Optimizing ventricular fibers: uniform strain or stress, but not ATP consumption, leads to high efficiency, American Journal of Physiology 283, pp. H1072-H1081, 2002.

[7] Shoucri, R.M., Theoretical study of the pressure-volume relation in left ventricle, American Journal of Physiology 260, pp. H282-H291, 1991.

[8] Shoucri, R.M., Possible clinical applications of the external work reserve of the myocardium, Japanese Heart Journal 35, pp. 771-787, 1994. 
[9] Shoucri, R.M., The active force of the myocardium (Chapter 3). Computer Simulations in Biomedicine, eds. H.Power \& R.T.Hart, Computational Mechanics Publications, Southampton, UK \& Boston, USA, pp. 19-26, 1995.

[10] Shoucri, R.M., The areas under ESPVR and the ventriculo-arterial coupling (Chapter 5). Simulations in Biomedicine IV, eds. H. Power, C.A. Brebbia \& J. Kenny, Computational Mechanics Publications, Southampton, UK \& Boston, USA, pp. 47-56, 1997.

[11] Shoucri, R.M., Studying the mechanics of left ventricular contraction, IEEE Engineering in Medicine and Biology Magazine 17(3), pp.95-101, 1998.

[12] Shoucri, R.M., Active and passive stresses in the myocardium, American Journal of Physiology 279, pp. H2519-H2528, 2000.

[13] Shoucri, R.M., The calculation of the intramyocardial stress, Technology and Health Care 10, pp. 11-22, 2002.

[14] Brandi, G. \& McGregor, M., Intramural pressure in the left ventricle of the dog, Cardiovascular Research 3, pp. 472-479, 1969.

[15] Shoucri, R.M., Pressure-volume relation in the right ventricle, Journal of Biomedical Engineering 15, pp.167-169,1993.

[16] Westerhof, N., Physiological hypotheses-intramyocardial pressure. A new concept, suggestions for measurement, Basic Research in Cardiology 85, pp.105-119,1990.

[17] Huisman, R.M., Sipkema, P., Westerhof, N. \& Elzinga, G., Comparison of models used to calculate left ventricular wall force, Medicine and Biological Engineering and Computing 18, pp. 133-144, 1980.

[18] Burns, J.W., Covell, J.W., Myers, R. \& Ross Jr., J., Comparison of directly measured left ventricular wall stress and stress calculated from geometric reference figures, Circulation Research 28, pp. 611-621, 1971.

[19] Yin, F.C.P., Ventricular wall stress, Circulation Research 49, pp. 829-842, 1981.

[20] Burkhoff, D. \& Sagawa, K., Ventricular efficiency predicted by an analytical model, American Journal of Physiology 250, R1021-R1027, 1986.

[21] Asanoi, H., Sasayama, S. \& Kamegama, T., Ventriculo-arterial coupling in normal and failing heart in humans, Circulation Research 65, pp. 483493, 1989.

[22] Mihailescu, L.S. \& Abel, F.L., Intramyocardial pressure gradients in working and working isolated cat hearts, American Journal of Physiology 266, pp. H1233-H1241, 1994. 
